\title{
A renormalization group approach to crowd psychology and inter-group coupling in social many-body systems
}

\author{
KIM Chul Koo ${ }^{1}$, LEE Kong-Ju-Bock ${ }^{2} \&$ CHUNG Myung-Hoon ${ }^{3 *}$ \\ ${ }^{1}$ Institute of Physics and Applied Physics, Yonsei University, Seoul 120-749, Korea; \\ ${ }^{2}$ Department of Physics, Ewha Woman's University, Seoul 120-750, Korea; \\ ${ }^{3}$ Department of Physics, Hongik University, Jochiwon, Choongnam 339-701, Korea
}

Received May 10, 2011; accepted July 10, 2011

\begin{abstract}
By adopting majority rule within a renormalization group approach, we can show that the strength of inter-group interaction in a social system either grows or shrinks monotonically with increasing group size, depending on the initial coupling strength of individuals in the group. This contrasts with the findings of previous studies in which the strength of the interaction grows with group size regardless of the initial strength. Our approach clearly demonstrates that the phenomena of crowd psychology, such as an extreme anti-reaction between different ethnic or ideological groups, could be a consequence of the many-particle nature of social systems when the initial strength of the interaction is larger than a critical value. The effect of neutral opinion holders is critically examined using the spin-one Ising model. The critical size of the population of neutral opinion holders, that can prevent crowd polarization, depends on the block spin rule.
\end{abstract}

renormalization, crowd psychology, block spin

Citation: Kim C K, Lee K, Chung M. A renormalization group approach to crowd psychology and inter-group coupling in social many-body systems. Chin Sci Bull, 2012, 57: 247-252, doi: 10.1007/s11434-011-4882-4

Crowds are known to act differently from individuals within the crowd. Several social science theories have been developed to explain crowd psychology. Although communities of people are extremely complex systems, they are expected to exhibit characteristics of many-body systems similar to other systems in nature. There have been many attempts by physicists to explain social phenomena using many-body theories in or out of the context of socio-physics [1-12]. One of the more fundamental problems is crowd psychology that results in extraordinary inter-community conflicts [13-19]. Because of this, studying the dependence of the coupling strength between different groups on the size of each group is interesting. These different groups could be characterized by different ideologies, religions, races, or opinions.

To describe interactions among individuals with the same or opposite opinions, the Ising model has been widely used [3,7-9]. More detailed analysis of social dynamics have been

*Corresponding author (email: mhchung@ hongik.ac.kr) shown to lead to models that are qualitatively similar to the two dimensional Ising model [20]. Because we are interested in variation of the inter-group attitude, it is necessary to consider a consensus-making process and a leading opinion of the community. The renormalization group (RG) approach, which is widely used in many-body problems, offers a natural theoretical framework for this purpose, because the two main concepts of the RG, the coarse graining and the block spin [21-23], can map onto the two social aspects, consensusmaking and leading opinion. In this paper, we adopt the RG approach to understand crowd psychology and inter-group conflicts by considering generalized Ising models.

\section{Model}

To simulate a group of individuals using a minimal model, we first assume that each individual holds one of two contrasting opinions and that the opinion of the individual $i$ as 
$S_{i}$ can be represented by $S_{i}= \pm 1$ although later we shall allow $S_{i}=0$ when we treat neutral-opinion holders. The social behavior favoring the presence of the same opinion in one's vicinity is reflected in the model by lowering the system's energy. Hence, the energy of our social system becomes lower when holders of the same opinion are the nearest neighbors than when holders of opposite opinions are the nearest neighbors. The above situation can be conveniently described by the Ising Hamiltonian,

$$
\begin{aligned}
\beta \mathcal{H} & =-\beta J \sum_{\langle i, j\rangle} S_{i} S_{j}-\beta H \sum_{i}^{N} S_{i} \\
& =-K \sum_{\langle i, j\rangle} S_{i} S_{j}-h \sum_{i}^{N} S_{i},
\end{aligned}
$$

where $\beta=1 /\left(k_{B} T\right)$ with $k_{B}$ the Boltzmann constant and $T$ the temperature. Various social backgrounds might be reflected as the thermal energy $k_{B} T$ in this model. The spin at site $i$ represents an individual among $N$ beings either with a positive $\left(S_{i}=+1\right)$ or a negative opinion $\left(S_{i}=-1\right)$. Note that the positive coupling constant $J>0$ lowers the system energy when two nearest neighbors have similar opinions and the magnetic field $H$ has the role of an external force-field that tends to polarize the opinions of the social groups in a desired direction. This model is essentially identical to a social impact model with nearest neighbor interaction [24]. Here, we note that most previous studies have focused mainly on the microscopic origin of the inter- and intra-group disliking (preference) strength, $K$ [13-19]. Using the prisoner's dilemma game, an extensive study of the differences between intergroup behavior and inter-individual behavior also focused on the microscopic mechanism of the phenomena [25,26]. Although a few phenomenological models have been proposed to understand phenomena related to changes in the interaction strength with group size, such as radical trends of union shops with different sizes [27], no serious effort has been made so far to understand the many-body nature of the mechanism underlying the changes in interaction with group size.

By introducing the concepts of coarse graining and block spins [21-23],

$$
S_{I} \equiv \frac{1}{\left|\bar{m}_{l}\right|} \frac{1}{l^{d}} \sum_{i \in I} S_{i}
$$

where

$$
\bar{m}_{l}=\frac{1}{l^{d}} \sum_{i \in I} S_{i} .
$$

Each block contains $l^{d}$ spins for a $d$-dimensional system. The effective Hamiltonian $\mathcal{H}_{l}$ is given by

$$
\beta \mathcal{H}_{l}=-K_{l} \sum_{\langle I, J\rangle}^{N l^{-d}} S_{I} S_{J}-h_{l} \sum_{I=1}^{N l^{-d}} S_{I},
$$

where $K_{l}$ is the effective coupling strength between opinions of subgroups and the effective social field is given by $h_{l}=h \bar{m}_{l} l^{d}$. We are interested in the behavior of $K_{l}$ when the size of subgroup $l$ expands continuously to understand the many-particle nature in the social phenomena of crowd psychology.

It has been shown that two dimensional Ising-like models successfully simulate social phenomena such as collective opinion formation and ghetto formation $[9,20]$. We believe that these two dimensional models represent good starting points to study social phenomena, although, in the future, studies of more complicated social networks are desirable $[6,28]$. It may also be argued that social particle systems are more similar to fluids than to lattices. However, it is well-known that fluids can be mapped to lattice gases [21], as shown by the disordered Ising model. The effect of disorder (neutral opinion) is discussed in detail below. Noting that the universality class and the global flow diagram are not dependent on lattice type, we choose a triangular lattice because many relevant results have been reported in previous studies $[21,22]$ and a new calculation in the presence of neutral opinion holders is also mathematically simple.

\section{Renormalization group approach}

\subsection{Without neutral opinion}

When there is no neutral opinion, the model is represented by the conventional two-dimensional Ising model. This case corresponds to a social system where each constituent is either polarized $\left(S_{i}=1\right)$ or anti-polarized $\left(S_{i}=-1\right)$. Now, we investigate the change of the inter-group coupling strength as a function of group size. Previously, the two dimensional Ising model of eq. (1) was solved exactly and perturbatively $[21,22]$. The rule used here to determine the block spin that corresponds to the opinion of a subgroup in the social system is the majority rule. Hence the block spin $S_{I}$ of block $I$ can be written as

$$
S_{I}=\operatorname{sign}\left\{S_{1}^{I}+S_{2}^{I}+S_{3}^{I}\right\},
$$

where $S_{j}^{I}$ is the $j$-th spin in the $I$-th triangular block. The majority rule does not allow an intermediate opinion, which means that a subgroup represents either $S_{I}=1$ or $S_{I}=$ -1 . More complicated situations for reaching consensus in general models have been recently discussed in the literatures $[29,30]$. Following the perturbation calculation for our simple system in [21], the fixed points are obtained as $K^{*}=0, \infty, 0.34$. The extremely weak (strong) coupling corresponds to a paramagnetic (ferromagnetic) fixed point. These results are in good agreement with Onsager's exact result, yielding a correct flow diagram for the two-dimensional Ising model. The result of this RG calculation can be interpreted in terms of crowd psychology as follows.

In the external social field, $h=0$, there exists a critical $K_{c}=0.34$ that determines whether the coupling strength between groups either grows or shrinks when the group size grows. However, the coupling strength will never reach the 
extreme fixed points, $\infty$ or 0 because of the finite size effect. When the initial coupling strength $K=\beta J$ for a particular ideology is smaller than the critical value, the intergroup coupling strength will decrease as the group size increases. By constrast, when the initial coupling strength is greater than the critical value, the coupling strength will increase with group size. This interpretation reflects aspects of crowd psychology that are seldom observed in individual psychology and arise from the many-body nature of social systems, especially when the initial coupling strength is greater than the critical value. We note that it is difficult to clearly define the coupling strength in social systems because there could be many factors affecting the coupling between individuals in crowds. In this sense, the above RG approach might contribute to determining the significance of the coupling strength by investigating whether any specific phenomenon is strengthened by an increase in group size.

An interesting example is provided by data collected from workers belonging to union shops of various sizes [27]. This study found a radical trend among workers by focusing on the proportion of workers saying that union policies are too moderate. This proportion increased from $64 \%$ to $81 \%$ as the average number of workers in the union shops increased from 60 to 1300. This supports the contention that workers in large union shops are more radical than those in small union shops. Hence the initial coupling strength between individual union members is expected to be larger than a critical value from the RG approach. Weakening of coupling strength with increasing group size has not drawn much attention among social scientists as such phenomena do not cause atypical effect on social systems. However, we expect many social systems do not show any special phenomena because of interactions between individuals that are weaker than a critical value. One possible example may be found in alumni organizations of large universities in which the coupling strength decreases with increasing group size. It should be noted that in previous phenomenological theories [27], the coupling strength always grows with increasing group size regardless of the initial strength.

A strong external social field $h$ results in a completely polarized opinion within the social system. This phenomenon could be observed in a group or society governed by an absolute rule. Recall that in eq. (1) the coupling strength $K$ and the social field $h$ are defined as the relative coupling and the relative field to the social thermal-energy $k_{B} T$. Any characteristics of society such as social culture, history, and customs could reflect the social thermal-energy although they are very difficult to define as measurable quantities. However, it is expected that a strong coupling $J$ itself could not induce any atypical collective phenomena as long as a coupling $K$, relative to the social background energy $k_{B} T$, is not sufficiently strong.

In the next subsection, we consider a group that could have members holding a neutral opinion and investigate the effect that neutral-opinion holders have in determining the coupling strength under majority rule.

\subsection{With neutral opinion}

The model considered in the previous subsection represents an idealized situation because only two opposing opinions were allowed. To simulate social systems more accurately, it is necessary to allow the presence of neutral opinions. In this subsection, we critically examine the effect that neutral opinion holders have on the coupling strength by allowing the Ising spin to have three options viz. $S_{i}=1,0,-1$. This spinone Ising model is described by the same Hamiltonian in eq. (1) with the modification of the possible spin values. Here, we assume that the site energies are equal for all possible spin values [31]. However, $S=0$ corresponding to a neutral opinion in the model causes ambiguity in fixing the block spin as given in eq. (2). We define the block spins by adopting the majority rule as follows:

$$
S_{I}=\left\{\begin{array}{cl}
1 & \text { if } S_{\max } / 2 \leqslant \sum_{i \in I} S_{i} \leqslant S_{\max } \\
0 & \text { if } S_{\min } / 2<\sum_{i \in I} S_{i}<S_{\max } / 2 \\
-1 & \text { if } S_{\min } \leqslant \sum_{i \in I} S_{i} \leqslant S_{\min } / 2
\end{array}\right.
$$

where $S_{\max }=\sum_{i \in I} 1$ is given by the number of sites inside the block, and $S_{\min }=-S_{\max }$. The majority rule is applied so that the block spin is neutral $\left(S_{I}=0\right)$ when neutral opinion holders form the majority in the block. Note that the block spin can also be zero when the numbers of polarized and anti-polarized opinion holders are equal. The equality signs for $S_{I}=1$ and -1 indicate that polarized opinions have an advantage in representing the opinion of the block when the numbers of neutral and polarized opinion holders are equal, as occurs in reality. However, such cases do not occur for the triangular lattice that we are considering.

Now, we perform the perturbative RG using the above block spin definition. First, we find that possible configurations of the original spins $\sigma_{I} \equiv\left\{S_{1}^{I}, S_{2}^{I}, S_{3}^{I}\right\}$ in triangular block $I$ can be classified by the block spin, $S_{I}$, and the number of neutral spins in the block, $n_{0}$, as listed in Table 1 . We begin by considering the effective Hamiltonian $\mathcal{H}^{\prime}\left\{S_{I}\right\}$ given by

Table 1 Classification for all possible configurations of Spins $\sigma_{I}$ in triangular block $I$ in terms of the block spin $S_{I}$ and the number of neutral spins $n_{0}$

\begin{tabular}{ccc}
\hline$S_{I}$ & $n_{0}$ & $\sigma_{I}$ \\
\hline 1 & 0 & $\downarrow \uparrow \uparrow, \uparrow \downarrow \uparrow, \uparrow \uparrow \downarrow, \uparrow \uparrow \uparrow$ \\
-1 & 1 & $0 \uparrow \uparrow, \uparrow 0 \uparrow, \uparrow \uparrow 0$ \\
& 0 & $\uparrow \downarrow \downarrow, \downarrow \uparrow \downarrow, \downarrow \downarrow \uparrow, \downarrow \downarrow \downarrow$ \\
0 & 1 & $0 \downarrow \downarrow, \downarrow 0 \downarrow, \downarrow \downarrow 0$ \\
& 1 & $0 \uparrow \downarrow, \uparrow 0 \downarrow, \uparrow \downarrow 0,0 \downarrow \uparrow, \downarrow 0 \uparrow, \downarrow \uparrow 0$ \\
& 2 & $00 \uparrow, 0 \uparrow 0, \uparrow 00,00 \downarrow, 0 \downarrow 0, \downarrow 00$ \\
\hline
\end{tabular}




$$
\exp \left(\mathcal{H}^{\prime}\left\{S_{I}\right\}\right)=\sum_{\left\{\sigma_{I}\right\}} \exp \left(\mathcal{H}\left\{S_{I}, \sigma_{I}\right\}\right)
$$

We estimate $\mathcal{H}^{\prime}$ using perturbation theory for the case $h=0$. The Hamiltonian can be written as

$$
\mathcal{H}=\mathcal{H}_{0}+V
$$

where

$$
\mathcal{H}_{0}=K \sum_{I} \sum_{i, j \in I} S_{i} S_{j},
$$

and the interaction between spins in different blocks is

$$
V=K \sum_{I \neq J} \sum_{i \in I, j \in J} S_{i} S_{j} .
$$

Using the following definition of the average

$$
\left\langle A\left(S_{i}\right)\right\rangle_{0} \equiv \frac{\sum_{\left\{\sigma_{I}\right\}} \exp \left(\mathcal{H}_{0}\left\{S_{I}, \sigma_{I}\right\}\right) A\left(S_{I}, \sigma_{I}\right)}{\sum_{\left\{\sigma_{I}\right\}} \exp \left(\mathcal{H}_{0}\left\{S_{I}, \sigma_{I}\right\}\right)},
$$

we find

$$
\exp \left(\mathcal{H}^{\prime}\left\{S_{I}\right\}\right)=\langle\exp (V)\rangle \sum_{\left\{\sigma_{I}\right\}} \exp \left(\mathcal{H}_{0}\left\{S_{I}, \sigma_{I}\right\}\right) .
$$

Using the cumulant expansion, we obtain

$$
\mathcal{H}^{\prime}\left\{S_{I}\right\}=M \log Z_{0}(K)+\langle V\rangle_{0}+O\left(V^{2}\right),
$$

where $M$ is the total number of blocks in the system and $Z_{0}(K)$ is the partition function for one block subject to a given value of $S_{I}$,

$$
Z_{0}(K)=\sum_{\left\{S_{1}, S_{2}, S_{3}\right\}} \exp \left(K\left(S_{1}^{I} S_{2}^{I}+S_{2}^{I} S_{3}^{I}+S_{3}^{I} S_{1}^{I}\right)\right) .
$$

From eqs. (6)-(14), we obtain

$$
\mathcal{H}^{\prime}\left\{S_{I}\right\}=M \log Z_{0}(K)+K^{\prime} \sum_{<I J>} S_{I} S_{J}+O\left(V^{2}\right) .
$$

With the configurations as listed in Table 1, the partition functions are written as

$$
Z_{0}\left(K, S_{I}= \pm 1\right)=3 \exp (-K)+3 \exp (K)+\exp (3 K),
$$

and

$$
Z_{0}\left(K, S_{I}=0\right)=6 \exp (-K)+7
$$

As can easily be seen from Figure 1, the interaction between spins in different blocks is given by

$$
\begin{aligned}
V & =\sum_{I \neq J} V_{I J}, \\
V_{I J} & =K S_{3}^{J}\left(S_{1}^{I}+S_{2}^{I}\right) .
\end{aligned}
$$

Therefore, we obtain

$$
\left\langle V_{I J}\right\rangle_{0}=2 K\left\langle S_{3}^{J}\right\rangle_{0}\left\langle S_{1}^{I}\right\rangle_{0}
$$

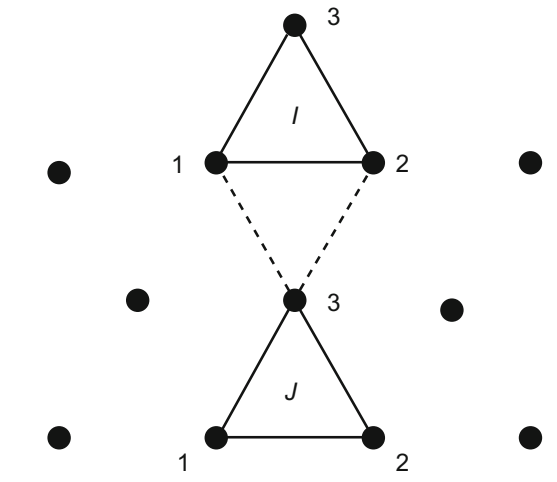

Figure 1 Interaction between nearest neighbor block spins to $O(V)$.

Following the same procedure for the two dimensional Ising model [21], we obtain

$$
\left\langle S_{3}^{J}\right\rangle=\Phi(K) S_{J}
$$

where

$$
\Phi(K)=\frac{\exp (-K)+2 \exp (K)+\exp (3 K)}{3 \exp (-K)+3 \exp (K)+\exp (3 K)} .
$$

This enables us to write

$$
\langle V\rangle_{0}=2 K \Phi(K)^{2} \sum_{<I, J\rangle}^{N} S_{I} S_{J},
$$

yielding the recursion relation for the fixed points,

$$
K^{\prime}=2 K \Phi(K)^{2} \text {. }
$$

From this recursion relation, we obtain the fixed points $0, \infty$, and $K_{1}^{*}=0.39$. The eigenvalue is obtained from $\Lambda_{K_{1}}=$ $\partial K^{\prime} /\left.\partial K\right|_{K_{1}^{*}} \sim 1.44$. Similarly the recursion relation for the external field is given by

$$
\delta h^{\prime}=3 \Phi(K) \delta h,
$$

and $\Lambda_{h_{1}}$ is found to be 2.12 .

The above calculation clearly indicates that the presence of neutral opinion holders increases the critical coupling strength $K_{c}$ from 0.34 to 0.39 thus making the social group more immune to mass polarization. The qualitative features of the flow diagram in the previous case of no neutral opinions are still valid here. Hence the inter-group coupling could grow or shrink as the size of the group increases, depending on the initial coupling strength compared with the critical value.

It is useful to examine the critical population size of the neutral opinion holders $n_{c}=1-p_{c}$, that can prevent flow to mass polarization. The value $p_{c}$ denotes the critical population size of polarized opinion holders. This percolation limit can be studied using a real space RG technique near the ferromagnetic fixed point [22]. The main idea is that at the percolation probability the system should be invariant under scaling by an arbitrary length. To calculate $p_{c}$, we first follow the majority rule defined in eq. (6) for the block spin as 
shown in Figure 2(a). The probability for a block up-spin is given by

$$
p^{\prime}=p^{3}+3 p^{2}(1-p)
$$

This recursion relation has two trivial fixed points at $p=0$ and $p=1$ and a nontrivial value at $p_{c}=0.5$. The understanding is that the system does not flow toward mass polarization as a whole when more than half of the population are neutral agents, $n>0.5$. Hence the neutral opinion holders first increase the critical coupling strength $K_{c}$ and eventually disrupt the mass polarization completely when the population of neutral opinion holders reaches the critical value, $n_{c}$.

In social systems, unlike physical systems such as alloys, block spin can be determined using various different rules $[29,30]$. To investigate whether different rules for defining block spins change the critical population size, we now adopt eq. (2) to determine block spins as shown in Figure 2(b). Carrying out the RG calculation as above, we obtain

$$
p^{\prime}=p^{3}+3 p^{2}(1-p)+3 p(1-p)^{2} .
$$

This recursion relation has two trivial fixed points at $p=0$ and $p=1$ and a nonphysical value of $p=2$. It shows that under the rule of eq. (2) for block spins, the system flows to the ferromagnetic fixed point regardless of the population of neutral agents only if $K$ is larger than $K_{c}$. This rather unrealistic situation originates from the peculiar nature of eq. (2). Eq. (2) stipulates that the presence of even a single polarized opinion holder can dominate over any number of neutral opinion holders. This situation could be equivalent to an existence of absolute dictatorship and explains why a small indoctrinated group can dominate over a much larger indifferent majority. It is interesting to note that eq. (2), a reasonable block spin rule for the Ising model describing many physical systems, becomes a dictatorial rule in social systems.

\section{Discussion}

Social phenomena of crowd psychology were analyzed using the renormalization group approach motivated from the many-body nature of social systems. We first allowed each individual in the social group to choose one opinion among two opposing positions. Next, we extended the analysis to three opinions to include a neutral opinion. For the RG

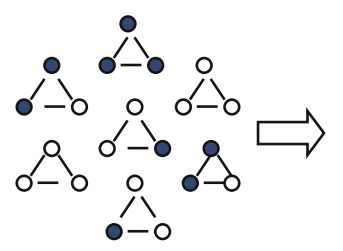

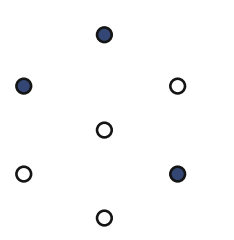

(a)

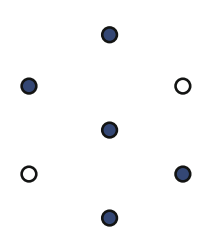

(b)
Figure 2 The one-step renormalization procedure for the triangular lattice. Polarized agents (up-spins) are marked as filled dots and neutral agents (zero spin) as empty dots. (a) The block spins defined following the majority rule of eq. (6). (b) The block spins defined following eq. (2). approach, majority rule is adopted to define block spins using the Ising model. Our main result is that the inter-group interaction in social systems could grow or shrink as the size of group increases depending on the initial coupling strength of individual in the group relative to critical value. This result is different from those of previous studies in which the coupling strength grows regardless of the initial strength. If the initial coupling of the individuals is weaker than the critical value, then the inter-group interaction shrinks resulting in no radical reaction of the social group. The critical population size of neutral opinion holders which can prevent mass polarization is also obtained using a real space renormalization approach and is shown to depend on the block spin rule.

The present theory can be used to test whether a newly emerging ideology or belief will grow stronger. In social systems, the absolute comparison of the coupling strength $K$ with $K_{c}$ is impossible and maybe meaningless. A useful test method might be be to compare the coupling strength at different group sizes. The 'soft' or 'rigid' behavior indicates the evolution of a new ideology. Small or medium scale persuasions or incentives to change beliefs or ideology can be used as a gauge to test the flow.

Although the present RG theory presents a consistent theoretical scheme for crowd psychology, several important refinements are needed to make the theory more realistic. One problem in the present theory is that the spins, which reflect personal opinions, are assumed quenched and thus timeindependent. In reality, persuasion can change opinions. Another problem arises from the increasing sophistication of social networks [32-34] in an internet-connected world [6,28]. Introduction of these factors in the model is expected to make the problem nonanalytic. However, we believe that it is imperative to include these aspects in future studies to better understand the complex nature of social systems.

This work was partially supported by the Max Planck Institute for the Physics of Complex Systems through a visiting program (to C.K.K.) and the Ministry of Education, Science and Technology of Korea through the National Research Foundation of Korea (2011-0008074 and 2010-00453) (to K.J.B.L.).

1 Auyang S. Foundation of Complex-System Theories. Cambridge: Cambridge University Press, 1998

2 Haken H. Advanced Synergetics. 2nd ed. Berlin: Springer, 1987

3 Callen E, Shapiro D. A theory of social imitation. Phys Today, 1974, July: 23-28

4 Helbing D, Keltsch J, Molnár P. Modelling the evolution of human trail systems. Nature, 1997, 388: 47-50

5 Weidlich W. Sociodynamics: A Systematic Approach to Mathematical Modeling in the Social Sciences. New Jersey: Harwood Academic, 2000

6 Albert R, Barabasi A. Statistical mechanics of complex networks. Rev Mod Phys, 2002, 74: 47-97

7 Aleksiejuk A, Holyst J, Stauffer D. Ferromagnetic phase transition in Barabasi-Albert networks. Physica A, 2002, 310: 260-266

8 Schelling T. Dynamic models of segregation. J Math Soc, 1971, 1: 143- 
186

9 Meyer-Ortmanns H. Immigration, integration and ghetto formation. Int J Mod Phys C, 2003, 14: 311-319

10 Bordogna C, Albano E. Theoretical description of teaching-learning processes: A multidisciplinary approach. Phys Rev Lett, 2001, 87: 118701

11 Mobilia M. Does a single zealot affect an infinite group of voters? Phys Rev Lett, 2003, 91: 028701

12 Slomczynski W, Zyczkowsk K. Penrose voting system and optimal quota. Acta Physica Polonica B, 2006, 37: 3133-3143

13 Levy S. Abstracts: Peace Science Society (International). In: ThirtyFourth North American Meetings, New Haven, Connecticut, October 27-29, 2000. Peace Economics, Peace Science and Public Policy, 2001, 7: Article 3

14 Hewstone M, Rubin R, Willis H. Intergroup-bias. Annu Rev Psychol, 2002, 53: 575-604

15 Bar-Tal D, Labin D. The effect of a major event on stereotyping: terrorist attacks in Israel and Israeli adolescents' perceptions of Palestinians, Jordanians and Arabs. Euro J Soc Psycho, 2001, 31: 265-280

16 Spruiell V. Crowd psychology and ideology: A psychoanalytic view of the reciprocal effects of folk philosophies and personal actions. Int $\mathrm{J}$ Psycho-Anal, 1988, 69: 171-178

17 Deffuant G, Amblard F, Weisbuch G, et al. How can extremism prevail? A study based on the relative agreement interaction model. J Artificial Societies and Social Simulation, 2002, 5: http://jasss.soc.surrey. ac.uk/5/4/1.html

18 Hegselmann R, Krause U. Opinion dynamics and bounded confidence: Models, analysis and simulation. J Artificial Societies and Social Simulation, 2002, 5: http://jasss.soc.surrey.ac.uk/5/3/2.html

19 Nye R. The Origins of Crowd Psychology: Gustave Le Bon and the Crisis of Mass Democracy in the Third Republic. London: Sage, 1975

20 Schweitzer F. Brownian Agents and Active Particles. New York: Springer, 2003. 359-371
21 Goldenfeld N. Lectures on Phase Transitions and the Renormalization Group. New York: Addison-Wesley, 1992. 257-268

22 Plischke M, Bergersen B. Equilibrium Statistical Physics. 2nd ed. Singapore: World Scientific, 1994. 228-236

23 Galam S. Social paradoxes of majority rule voting and renormalization group. J Stat Phys, 1990, 61: 943-951

24 Kacperski K, Holyst J. Phase transitions and hysteresis in a cellular automata-based model of opinion formation. J Stat Phys, 1996, 84: 169-189

25 Insko C, Schopler J, Hoyle R, et al. Individual-group discontinuity as a function of fear and greed. J Personality Social Psychol, 1990, 58: 68-79

26 Schopler J, Insko C. In: Stroebe W, Hewstone M, eds. European Review of Social Psychol. Chichester: Wiley, 1992. 121-152

27 Coleman J. Introduction to Mathematical Sociology. London: CollierMacmillan, 1964. 283-287

28 Wassermann S, Faust K. Social Network Analysis: Methods and Applications. Cambridge: Cambridge University Press, 1994

29 Stauffer D. The Monte Carlo method in the physical sciences: Celebrating the 50th anniversary of the metropolis algorithm. AIP Conf Proc, 2003, 690: 147-155

30 Sznajd-Weron K, Sznajd J. Opinion evolution in closed community. Int J Mod Phys C, 2000, 11: 1157-1165

31 Buyukkilic F, Demirhan D, Tirnakli U. Generalization of the mean-field Ising model within tsallis thermostatistics. Physica A, 1997, 238: 285294

32 Fu F, Chen X, Liu L, et al. Social dilemmas in an online social network: The structure and evolution of cooperation. Phys Lett A, 2007, 371: $58-64$

33 Andrade R R S, Miranda J G V, Pinho S T R, et al. Measuring distances between complex networks. Phys Lett A, 2008, 372: 5265-5269

34 Hu H, Wang X. Evolution of a large online social network. Phys Lett A, 2009, 373: 1105-1110

Open Access This article is distributed under the terms of the Creative Commons Attribution License which permits any use, distribution, and reproduction in any medium, provided the original author(s) and source are credited. 